\title{
Delineating nonmelanoma skin cancer margins using terahertz and optical imaging
}

\author{
Anna N. Yaroslavsky ${ }^{1,3^{*}}$, Cecil Joseph ${ }^{1}$, Rakesh Patel ${ }^{1}$, Alona Muzikansky ${ }^{2}$, Victor A. Neel ${ }^{3}$, \\ and Robert H. Giles ${ }^{1}$ \\ ${ }^{1}$ Department of Physics and Applied Physics, University of Massachusetts, Lowell, 1 University Ave., Lowell, MA 01854 \\ ${ }^{2}$ MGH Biostatistics Center, Massachusetts General Hospital, 50 Staniford Street, Boston, MA 02114 \\ ${ }^{3}$ Department of Dermatology, Massachusetts General Hospital, 50 Staniford Street, Boston, MA 02114 \\ * e-mail: Anna Yaroslavsky@uml.edu
}

\begin{abstract}
We evaluated terahertz reflectance imaging and a combination of terahertz and optical techniques for delineating nonmelanoma skin cancers. After imaging, each terahertz pixel of each specimen was classified as cancerous or normal using a previously determined threshold. Subsequently, cancerous terahertz pixels were reevaluated with polarization-sensitive optical technique. This additional information enabled us to re-classify each cancerous terahertz pixel using morphological appearance of the structures in the optical images. If the structures in the terahertz pixel appeared normal, the pixel was considered normal. The generalized linear mixture model was implemented to determine the sensitivity and specificity of each method. The model tested the probability of each terahertz pixel being diagnosed as a match with histology. For terahertz imaging alone, the sensitivity and specificity were $82 \%$ and $94 \%$, respectively. For multimodal imaging, the sensitivity and specificity increased to $96 \%$ and $99 \%$, respectively. Multimodal terahertz-optical imaging has potential for the intraoperative assessment of tumor margins. (C) 2017 Journal of Biomedical Photonics \& Engineering.
\end{abstract}

Keywords: imaging, cancer.

Paper \#3170 received 8 Mar 2017; revised manuscript received 24 Mar 2017; accepted for publication 25 Mar 2017; published online 29 Mar 2017. doi: 10.18287/JBPE17.03.010301. [Special Issue. Years in Biophotonics: 70th Anniversary of Prof. A.V. Priezzhev].

\section{References}

1. M. Al-Arashi, E. Salomatina, and A. N. Yaroslavsky, "Multimodal Confocal Microscopy for the Detection of Nonmelanoma Skin Cancers," Las. Surg. Med. 39(9), 706-15 (2007).

2. A. N. Yaroslavsky, J. Barbosa, V. Neel, C. DiMarzio, and R. R. Anderson, "Combining multi-spectral polarized-light imaging and confocal microscopy for localization of nonmelanoma skin cancer,” J Biomed Opt 10(1), 014011 (2005).

3. T. Gambichler, A. Orlikov, R. Vasa, G. Moussa, K. Hoffmann, M. Stücker, P. Altmeyer, and F. G. Bechara, "In vivo optical coherence tomography of basal cell carcinoma," J Dermatol Sci 45(3), 167-173 (2007).

4. J. Welzel, “Optical coherence tomography in dermatology: a review," Skin Res Tech 7(1), 1-9 (2001).

5. S. L. Jacques, J. R. Roman, and K. Lee, "Imaging superficial tissues with polarized light,” Las. Surg. Med. 26(2), 119-129 (2000).

6. A. N. Yaroslavsky, V. A. Neel, and R. R Anderson, "Demarcation of nonmelanoma skin cancer margins in thick excisions using multispectral polarized light imaging," J Inv. Derm. 121(2), 259-266 (2003).

7. A. N. Yaroslavsky, V. Neel, and R. R. Anderson, "Fluorescence polarization imaging for delineating nonmelanoma skin cancers," Opt. Lett. 29(17), 2010-2012 (2004).

8. A. N. Yaroslavsky, E. V. Salomatina, V. Neel, R. Anderson, and T. Flotte, "Fluorescence polarization of tetracycline derivatives as a technique for mapping nonmelanoma skin cancers," J Bio. Opt. 12(1), 014005 (2007).

9. Z. Tannous, M. Al-Arashi, S. Shah, and A. N. Yaroslavsky, "Delineating Melanoma Using Multimodal Polarized Light Imaging," Las. Surg. Med. 41(1), 10-16 (2009). 
10. C. A. Lieber, S. K. Majumder, D. L. Ellis, D. D. Billheimer, and A. Mahadevan-Jansen, "In vivo nonmelanoma skin cancer diagnosis using Raman microspectroscopy," Las. Surg. Med. 40(7), 461-467 (2008).

11. J. Choi, J. Choo, H. Chung, D. G. Gweon, J. Park, H. J. Kim, S. Park, and C. H. Oh, "Direct observation of spectral differences between normal and basal cell carcinoma (BCC) tissues using confocal Raman microscopy,” Biopolymers 77(5), 264-272 (2005).

12. R. M. Woodward, B. E. Cole, V. P. Wallace, R. J. Pye, D. D. Arnone, E. H. Linfield, and M. Pepper, "Terahertz pulse imaging in reflection geometry of human skin cancer and skin tissue," Phys. Med. Bio. 47(21), 3853-3863 (2002).

13. E. Pickwell, B. E. Cole, A. J. Fitzgerald, M. Pepper, and V. P. Wallace, "In vivo study of human skin using pulsed terahertz radiation,” Phys. Med. Bio. 49(9), 1595-1607 (2004).

14. V. P. Wallace, A. J. Fitzgerald, E. Pickwell, R. J. Pye, P. F. Taday, N. Flanagan, and T. Ha, "Terahertz pulsed spectroscopy of human basal cell carcinoma," App. Spectr. 60(10), 1127-1133 (2006).

15. B. Fan, V. A. Neel, A. N. and Yaroslavsky, "Multimodal Imaging for Nonmelanoma Skin Cancer Margin Delineation,” Las. Surg. Med., to be published (2016).

16. C. S. Joseph, R. Patel, V. Neel, R. H. Giles, and A. N. Yaroslavsky, "Imaging of ex vivo nonmelanoma skin cancers in the optical and terahertz spectral regions," J. Biophoton. 7(5), 295-303 (2012).

17. E. Salomatina, A. Muzikansky, V. Neel, and A. N. Yaroslavsky, "Multimodal optical imaging and spectroscopy for the intraoperative mapping of nonmelanoma skin cancer,’ J Appl Phys 105(10), 102010-102017 (2009).

\section{Introduction}

More people are diagnosed with nonmelanoma skin cancer (NMSC) in the United States yearly than with all other cancers combined. Most of these patients undergo traditional excisional surgery, which is performed without objective intraoperative tumor margin evaluation. Standard surgery uses the visual size and differentiation of the lesion to determine surgical margins. Resected specimens are forwarded to pathology, which examines only up to $2 \%$ of the surgical margin. A minority of the patients $(\sim 25 \%)$ are treated using Mohs Micrographic Surgery (MMS), which uses histopathological frozen sections and evaluates $100 \%$ of the margins during the surgical procedure. The MMS surgery continues till the margins are clear of cancer. MMS achieves up to $99 \%$ cure rates and significantly limits the morbidity compared to nonMohs procedures. However, MMS is not available at all dermatology facilities. Also, it is not considered appropriate for treating $75 \%$ of non-melanoma skin cancers. Thus, several techniques are currently under investigation for intraoperative demarcation of cancer margins. These techniques include confocal microscopy $[1,2]$, optical coherence tomography (OCT) [3, 4], endogenous and exogenous polarization and fluorescence anisotropy imaging [5-9], and Raman Spectroscopy [10, 11]. Terahertz pulsed imaging has also been investigated for skin cancer treatment [12-15].

Recently, we have proposed a combination of continuous-wave terahertz imaging (CWT) and polarization sensitive reflectance optical imaging (PLI) as a multimodal approach to intraoperative delineation of nonmelanoma skin cancers [15, 16]. The primary reason for interest in cancer imaging at terahertz frequencies is intrinsic contrast. However, due to the relatively long wavelengths, spatial resolution of terahertz frequencies is insufficient for visualization of tissue morphology. Conversely, optical imaging enables higher resolution but often lacks contrast for rapid and reliable tumor delineation. Therefore, in this contribution we have experimentally tested and statistically evaluated a multi-modal CWT and PLI approach for nonmelanoma skin cancer demarcation. We imaged ten fresh specimens of NMSC using both the PLI and CWT imaging systems. We utilized terahertz data obtained in our previous study [16] to establish a threshold for cross-polarized terahertz reflectance signals between normal and tumor tissue. Using the established threshold we delineated tumor margins in CWT images acquired in this study. Then we re-evaluated regions outlined as cancerous in CWT using PLI. Finally, we statistically evaluated and compared the imaging results yielded by CWT and a combination of CWT and PLI using H\&E histology as a gold standard.

\section{Materials and Methods}

\subsection{Tissue Handling}

Fresh discarded tissue specimens were obtained from the Massachusetts General Hospital within 40 minutes after surgeries under an IRB approved protocol. In total, seven squamous carcinomas and three basal cell carcinomas were imaged for this study. The samples came from 7 male and 3 female patients following Mohs micrographic surgery. The patient ages ranged from 53 to 77 . The specimen sizes varied approximately between $6 \mathrm{~mm}$ and $20 \mathrm{~mm}$ in the lateral dimensions and from $3 \mathrm{~mm}$ to $7 \mathrm{~mm}$ in depth. The respective data are summarized in Table 1. All cancer specimens included tumor and normal regions. Upon arrival to the Advanced Biophotonics Laboratory, the samples were quickly mounted in an aluminum sample holder with a quartz window (z-cut quartz for $\mathrm{THz}$ and amorphous quartz for optical). Mounted specimens were imaged with the optical system and terahertz systems. The dermal side of the tissue was imaged and the sample was backed by gauze soaked in phosphate buffered saline (PBS) to avoid dehydration during the 
Table 1 Nonmelanoma Skin Cancer Specimens. ${ }^{a}$

\begin{tabular}{ccccc} 
Sample \# & Gender & Age & Diagnosis & Size (mm) \\
\hline 1 & F & 74 & SCC & $22 \times 13 \times 5$ \\
\hline 2 & F & 74 & SCC & $23 \times 12 \times 6$ \\
\hline 3 & M & 56 & SCC & $13 \times 13 \times 4$ \\
\hline 4 & M & 71 & BCC & $9 \times 4 \times 3$ \\
\hline 5 & M & 73 & SCC & $22 \times 13 \times 7$ \\
\hline 6 & M & 73 & SCC & $22 \times 12 \times 5$ \\
\hline 7 & M & 66 & SCC & $17 \times 15 \times 4$ \\
\hline 8 & F & 74 & BCC & $20 \times 11 \times 5$ \\
\hline 10 & M & 77 & SCC & $18 \times 17 \times 3$ \\
\hline M & 53 & BCC & $16 \times 12 \times 3$
\end{tabular}

${ }^{a}$ BCC - Basal Cell Carcinoma, SCC - Squamous Cell Carcinoma

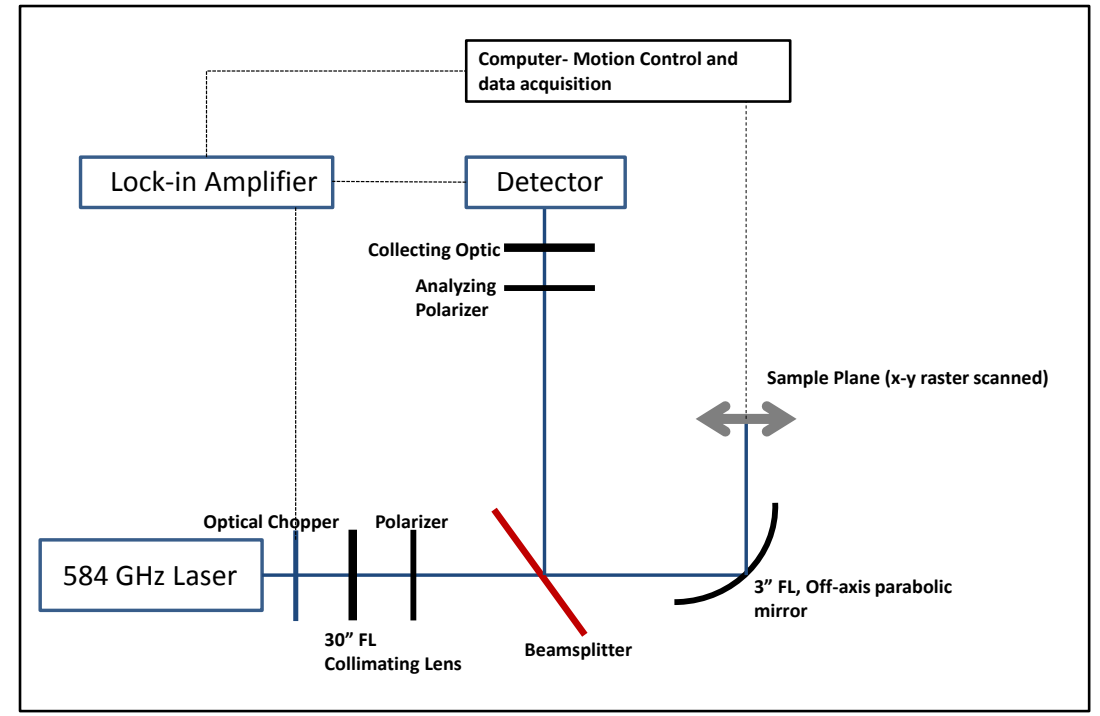

Fig. 1 Terahertz Imaging System.

measurements. Following imaging, the specimens were processed for frozen $\mathrm{H} \& \mathrm{E}$ histopathology from approximately the same en face planes that were imaged.

\subsection{Terahertz imaging}

The CW Terahertz system used for this experiment has been described in detail previously [16]. A lay-out is presented in Figure 1. A custom designed and built $\mathrm{CO}_{2}$ optically pumped far-infrared (FIR) gas laser was used as the source. The system operational frequency was $584 \mathrm{GHz}(513 \mu \mathrm{m})$. The vertically polarized beam had an output power of $10 \mathrm{~mW}$. For detecting the reflected signal we used a liquid Helium cooled silicon bolometer manufactured by IR Labs (Tucson, AZ). The detector's noise equivalent power (NEP) was $1.13 \times 10^{-13} \mathrm{~W} / \sqrt{\mathrm{Hz}}$, responsivity was $2.75 \times 10^{5} \mathrm{~V} / \mathrm{W}$, response time was 5 $\mathrm{ms}$ and gain was 200. System optics focused the beam down onto the imaging plane and the samples were raster scanned across the beam spot using an automated $\mathrm{x}-\mathrm{y}$ stage. The beam full width at half max (FWHM) at the focus was measured to be $0.67 \mathrm{~mm}$. The normally incident reflectance signal was registered. Prior to entering the detector, the signal passed through an analyzing wire-grid polarizer. For these experiments, the analyzer was set to collect the cross-polarized remittance. An optical chopper was used to modulate the $\mathrm{THz}$ beam and a lock-in-amplifier was used to collect data. The dwell time per point in each image was about $150 \mathrm{~ms}$ and the system's signal-to-noise ratio (SNR) was measured to be $65 \mathrm{~dB}$. The motion control and data acquisition was synchronized using a LabView ${ }^{\circledR}$ program.

After acquisition, the images were calibrated against the full-scale return of a flat front surface gold mirror. Cross-polarized terahertz reflectance image data was normalized using the formula: 


$$
R_{T H z}=\frac{I_{\text {Meas }}}{I_{\text {full scale }}} \times 100 \%
$$

where $R_{T H z}$ is the normalized terahertz cross-polarized reflectivity value, $I_{\text {Meas }}$ was the calibrated reflectivity of the pixel, and $I_{\text {full scale }}$ is the full-scale return from a flat front-surface gold mirror.

We determined a threshold reflectivity for cancer using the data from our previous study [16], where we measured the mean normalized terahertz reflectivity values for tumor and non-tumor structures by comparison with frozen $\mathrm{H} \& \mathrm{E}$ stained histopathology. Normalized terahertz reflectivity value of 0.17 , which corresponds to the mean tumor normalized reflectivity plus one standard deviation, was selected as a threshold, i.e., each terahertz pixel $(0.5 \mathrm{~mm} \times 0.5 \mathrm{~mm})$ was classified as normal if its normalized reflectivity was greater than 0.17, and as tumor if its normalized reflectivity was less than 0.17 . Based on this classification, acquired terahertz images were converted to binary black and white maps of tumor and normal tissue.

\subsection{Optical Imaging}

An optical imaging device is shown in Figure 2. A xenon arc lamp (Lambda LS, Sutter, Novanto, CA) combined with a narrow bandpass filter centered at 440 $\mathrm{nm}$, with full width at half maximum of $10 \mathrm{~nm}$, was used as the illuminator. The samples were illuminated at the oblique angle of approximately $50^{\circ}$ from the sample plane. The geometry was optimized to provide uniform illumination of the imaged field. Images were acquired using a CCD camera (CoolSnap Monochrome Photometrics, Roper Scientific, Tucson, AZ) with an attached 0.5X Rodenstock lens. To enable polarization imaging, linearly polarizing filters (Meadowlark Optics, Frederick, CO) were placed in pathways of the light incident on the sample and light collected by the camera. Reflectance co- and cross-polarized images were acquired with an analyzing polarizer oriented parallel and perpendicular to the polarization of the incident light, respectively. The system's field of view was $2.8 \mathrm{~cm} \times 2.5 \mathrm{~cm}$, and a lateral resolution was approximately $15 \mu \mathrm{m}$. Optical wide-field images were acquired in less than a half a second.

Optical polarization images were processed using the following formula:

$$
P L I=I_{c o}-G * I_{\text {cross }},
$$

where $P L I$ is the polarized light image, $I_{c o}$ is the copolarized image, $I_{\text {cross }}$ is the cross-polarized image, and $G$ is a calibration factor ( 0.99 for the presented system) which accounts for the bias in the system towards either polarization. The image acquisition and processing were performed utilizing Metamorph (Molecular Devices LLC., Sunnyvale, CA).

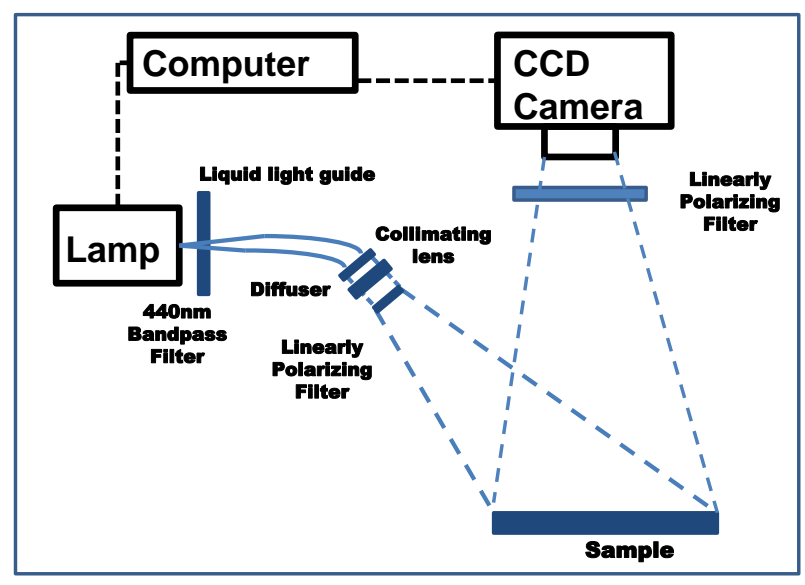

Fig. 2 Optical Imaging System.

\subsection{Histopathology}

En face frozen H\&E histopathology was processed from the tissue samples. After imaging, several $5 \mu \mathrm{m}$ thick slices were cut from the imaged specimens at the approximate planes that were imaged. Then the tissue sections were transferred to glass slides and stained with hematoxylin and eosin (H\&E). Histopathology slides were digitized using a Zeiss Axioscope microscope (Zeiss, Germany, 5X objective lens, NA 0.13). The terahertz and optical images were compared with corresponding digitized histopathology.

\subsection{Data Analysis and Statistics}

For comparative evaluation of the results yielded by terahertz versus combined terahertz and optical imaging we overlaid the terahertz, optical, and histopathological images [8]. To assess the sensitivity and specificity of cross-polarized continuous-wave terahertz imaging and to quantify the enhancement to sensitivity and specificity provided by combining optical polarized light imaging with terahertz imaging we devised statistical analysis to overcome the limited number of specimens that were available for the project. We have constructed generalized linear mixture model. This model treats the tumor specimens as repeat experiments. Each terahertz pixel of each specimen (lateral size of $0.5 \mathrm{~mm} \times 0.5 \mathrm{~mm}$ ) was considered as an individual statistical data point. Then we determined the probability of each terahertz pixel being diagnosed as a match with histology as a function of the method (terahertz or combined optical/terahertz). For sensitivity tests, only the pixels, which were defined as cancer in histopathology were employed, whereas for specificity, only the pixels that were defined as normal were used. The number of statistical data points varied between specimens and ranged from 8 to 474 (as presented in table 2). We derived a sensitivity and specificity score for each specimen using repeated observation model with correlation structure taking into account the inherit correlation of adjacent statistical data points. We also determined the overall global scores across all 10 specimens. In addition, we calculated a p-value for the 
difference between the terahertz and combined terahertz-optical methods per each sample using McNemar's test.

\section{Results and Discussion}

Example images of a sample with residual squamous cell carcinoma (SCC) are presented in Figure 3. False color and thresholded cross-polarized terahertz images are shown in Figures 3 a and b, respectively. In Figure 3 $\mathrm{b}$, terahertz image is presented as black and white binary map, where black color corresponds to the areas classified as tumor with normalized terahertz reflectivity values lower than 0.17 . Optical cross-polarized and PLI images, as well as corresponding digitized $\mathrm{H} \& \mathrm{E}$ histopathology are presented in Figures $3 \mathrm{c}$, d, and e, respectively. The cancer is outlined with a dashed line in Figure 3 e. Tumor areas can be clearly identified in terahertz (Figs. 3 a and b) and optical images (Figs. 3 c and $\mathrm{d}$ ). However, even though the contrast of the terahertz image in Figure $3 \mathrm{a}$ is higher, as compared to optical images, the actual shape of the tumor is better visualized in the optical images (Figures $3 \mathrm{c}$ and $\mathrm{d}$ ), which correlates well with histology. It can also be appreciated that several black areas, which indicate tumor in the thresholded terahertz image, along the rim of the specimen (Figure $3 \mathrm{~b}$ ) are not confirmed by the gold standard of histopathology (Figure 3 e). This indicates that some terahertz pixels were misclassified.

To enable higher resolution inspection of these pixels, we examined corresponding areas in optical images outlined with squares in Figure 3 and compared them with respective areas in histopathology. The results are presented in Figures 4 and 5. The false positive area at the bottom of the image in Figure $3 \mathrm{~b}$ is outlined with solid line and magnified in Figure 4. Detailed examination of optical superficial PLI (Figure 4 a) and cross-polarized images (Figure 4 b) reveals normal skin structures, such as epidermis (dotted

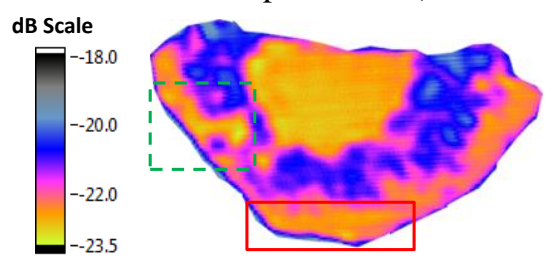

(a) arrow), dermis (solid arrow), hair follicles (dash and dotted arrows) and fat (dashed arrow). Both optical images correlate closely with respective histopathology shown in Figure $4 \mathrm{c}$.

The area to the left of the cancer nodule that shows up as false positive, is outlined with a dashed square in Figure 3, and is magnified in Figure 5. Similarly, it does not present the structural loss that indicates tumor in the optical images. It reveals normal epidermis (dotted arrow), dermis (solid arrow), fat (dashed arrow) and a lot of hair follicles (dash and dotted arrow).

Low terahertz reflectivity at the epidermal borders of the specimen (Figure $3 \mathrm{a}$ and $\mathrm{b}$ ) can be explained by uneven thickness of the excisions, which are usually thinner towards epidermal edge. The gaps between tissue and cover slip are filled with saline solution, which increases absorption of terahertz radiation.

In contrast with terahertz images, optical images exhibit sufficient resolution, similar to that of histopathology. They enable identification of false positive and false negative spots. In this manner, all false negative and false positive pixels in the terahertz image were reclassified by a qualitative comparison with the overlaid PLI and cross-polarized optical images. If a region demarcated by terahertz imaging as tumor was also identified by the optical images as an area which exhibited a loss of normal morphological structure, it was classified as tumor. If, on the other hand, the region did not show a loss of structure and exhibited features of normal skin components, such as epidermis, dermis, subcutaneous fat, hair follicles, etc., it was reclassified as normal. Thus using the optical imaging we were able to convert false positive pixels to true negatives and false negatives to true positives based on qualitative high resolution morphological information.

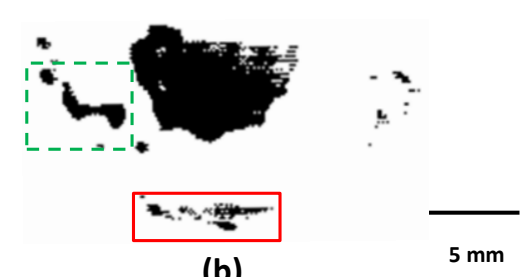

(b)

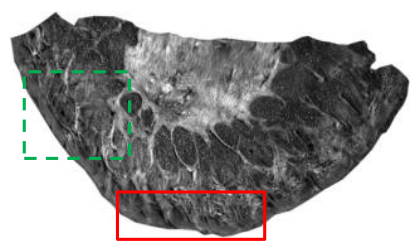

(c)

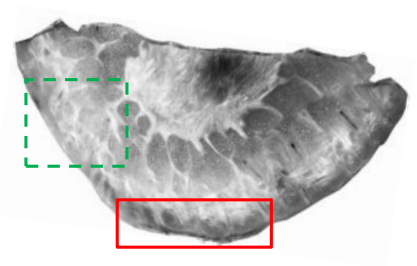

(d)

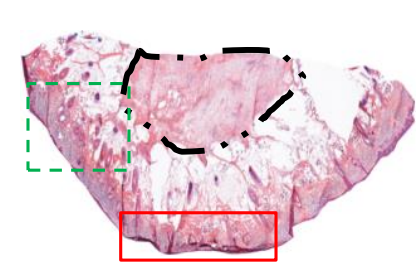

(e)

Fig. 3 Images of a skin sample with squamous cell carcinoma. The areas within solid and dashed squares are magnified in figures 4 and 5, respectively. (a) Terahertz image. (b) Thresholded terahertz image. (c) Superficial polarized light optical image (PLI). (d) Cross-polarized optical image. (e) Histopathology with tumor, outlined by dash-and dot line. 


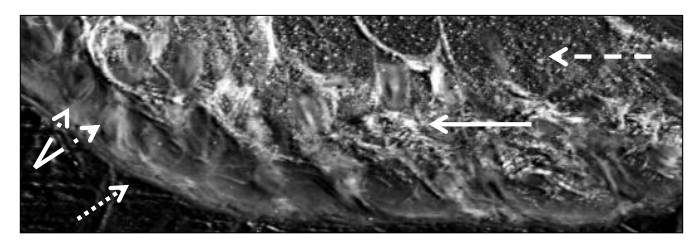

(a)

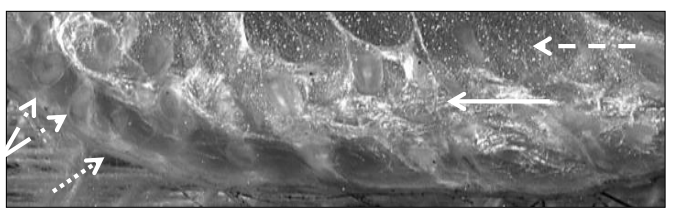

(b)

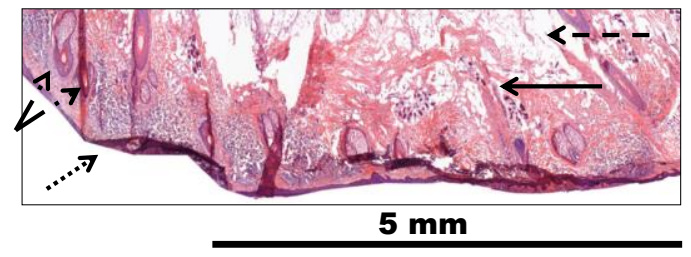

(c)

Fig. 4 Images of a skin specimen with SCC. Solid arrow points to collagen, dashed arrow to fat, dotted arrow to epidermis, dash and dotted arrows to hair follicles. (a) Superficial polarized light optical image (PLI). (b) Cross-polarized optical image. (c) Histopathology.

Our results, shown in Figures 3-5, demonstrate that terahertz and optical images offer complementary information that can potentially be used for the accurate assessment of skin cancer margins. In particular, terahertz imaging provides intrinsic contrast, which helps to locate areas suspicious for cancer without extrinsic contrast agents. Our proposed method of quantitative analysis of the terahertz images relies on thresholding of normalized cross-polarized remittance from the sample to highlight areas suspicious for tumor. Thus, areas of low cross-polarized reflectivity may indicate cancerous areas, while higher reflectivity values indicate normal tissue. However, as can be seen in Figure 3, simply applying a threshold to the image is insufficient for margin delineation, false positive (dark areas near sample edges) and false negatives (white areas within tumor boundary) also occur and the resolution of the terahertz image is insufficient to make out any morphological information that could resolve this. Optical imaging has an advantage of higher resolution that helps with accurate assessment of tissue morphology in the areas highlighted by terahertz imaging. As can be appreciated in Figures 4 and 5, combining information from the optical and terahertz images allows for reduction and in some cases, complete elimination of false positives and false negatives by re-examining areas characterized by low reflectivity in terahertz image. Thus the terahertz image serves as a beacon to localize potential tumor sites, while optical imaging maps tissue morphology and is used for accurate and precise margin delineation, as well as for eliminating artifacts and false positives. A characteristic feature of the proposed multimodal technique, potentially important for future real-time implementation, is a considerable economy of time and computational resources achieved through selective optical analysis of only cancer-suspicious terahertz pixels. (a)

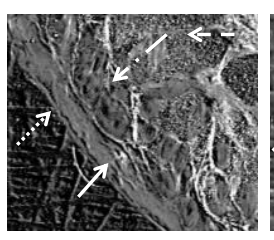

(b)

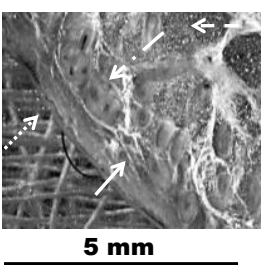

(c)

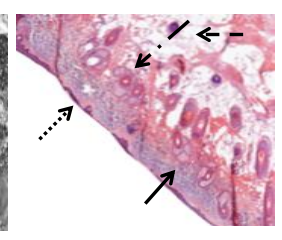

Fig. 5 Images of a skin specimen with SCC. Solid arrow points to collagen, dashed arrow to fat, dotted arrow to epidermis, dash and dotted arrow to hair follicles. (a) Superficial polarized light optical image (PLI). (b) Cross-polarized optical image. (c) Histopathology.

Table 2 Sensitivity and Specificity of Terahertz and Terahertz + Optical Methods.

\begin{tabular}{ccccccc}
\multirow{2}{*}{$\begin{array}{c}\text { Specimen } \\
\#\end{array}$} & \multicolumn{2}{c}{$\begin{array}{c}\text { \# of Statistical Data } \\
\text { Points }\end{array}$} & \multicolumn{2}{c}{ Terahertz Imaging } & \multicolumn{2}{c}{$\begin{array}{c}\text { Terahertz + Optical } \\
\text { Imaging }\end{array}$} \\
\cline { 2 - 7 } & Sensitivity & Specificity & Sensitivity & Specificity & Sensitivity & Specificity \\
\hline 1 & 150 & 474 & 90.67 & 97.47 & 100 & 99.16 \\
\hline 2 & 184 & 440 & 77.17 & 94.09 & 98.91 & 100 \\
\hline 3 & 132 & 444 & 100 & 97.75 & 100 & 99.1 \\
\hline 4 & 8 & 136 & 100 & 98.53 & 100 & 100 \\
\hline 5 & 142 & 298 & 61.97 & 97.99 & 94.37 & 100 \\
\hline 6 & 78 & 468 & 66.67 & 92.74 & 87.18 & 96.15 \\
\hline 7 & 104 & 536 & 92.31 & 90.3 & 94.23 & 99.25 \\
\hline 8 & 68 & 252 & 97.06 & 96.03 & 97.06 & 99.21 \\
\hline 9 & 218 & 258 & 56.88 & 90.7 & 97.25 & 97.67 \\
\hline 10 & 138 & 318 & 78.26 & 88.05 & 91.3 & 94.97 \\
\hline Overall & & & 82.1 & 94.4 & 96.03 & 98.55
\end{tabular}


For statistical evaluation, we have determined the sensitivity and specificity of terahertz imaging alone. Then the areas positive for cancer in terahertz images were re-evaluated based on the correlation between optical images and histopathology. The results of statistical analysis for the sample presented in Figures 3, 4 and 5 , show that the sensitivity and specificity of terahertz imaging alone is $82 \%$ and $94 \%$, respectively. Combined optical and terahertz image analysis improved the sensitivity and specificity to $96 \%$ and 99\%, respectively. McNemar's test confirmed that the two methods, i.e. terahertz and combined optical terahertz imaging, are significantly different with a $\mathrm{p}<0.0001$. In total we have imaged and analyzed 10 samples. The results of statistical analysis performed for each sample are presented in table 2 . In all the cases investigated, the combination of optical and terahertz imaging yielded highly significant improvement on the results of terahertz imaging alone. These results are comparable to those yielded by contrast enhanced polarization optical imaging. [6-8, 17]. However, the technique presented in this paper does not require the use of exogenous contrast agents, which could potentially cause adverse phenomena such as allergic reactions.

The analysis of the results of the entire set of images collected in the study using a generalized linear mixture model, which took into consideration the inherit correlation within each sample and the pairing of the observations, demonstrated that the difference between terahertz and combined optical / terahertz imaging was statistically significant with $p$-value $<0.0001$. It has also confirmed that the combined optical and terahertz imaging yielded more predictive results, i.e., provided better correlation with the gold standard of histopathology.

It should be noted that in its current implementation the terahertz imaging system cannot be deployed in clinic. However, we plan to develop a combined terahertz and optical system for imaging surgical bed during skin cancer surgeries that would offer effective tumor delineation without the need for exogenous contrast agents.

\section{Summary}

In this paper we presented experimental evaluation and statistical comparison of two methods, i.e., crosspolarized continuous-wave terahertz imaging and multimodal polarization-sensitive optical and terahertz approach, which can be termed on-demand optical-overterahertz imaging, for the intraoperative ex vivo delineation of NMSC.

We have tested our approach to combining diagnostic information yielded by the terahertz and optical imaging, where terahertz image is used to reveal potential tumor sites, while morphological analysis of the optical images is confined to the areas highlighted by the terahertz approach. This allows for effective elimination of the false positives and leads to an optimized cancer delineation procedure achieved through selective optical analysis of only cancersuspicious terahertz pixels.

We determined the sensitivity and specificity of the terahertz only and multimodal methods using generalized linear mixture model. To the best of our knowledge, this is the first report of the sensitivity and specificity of the terahertz and multimodal terahertz-PLI imaging for skin cancer demarcation. Statistical analysis revealed that combined optical PLI and terahertz imaging is significantly different and yields significant improvement of sensitivity and specificity, as compared to the terahertz imaging alone.

Our results indicate that combined on-demand optical-over-terahertz imaging of skin cancer has the potential to provide accurate and rapid intraoperative margin delineation during skin cancer removal, potentially eliminating the need for histology processing at every stage of the surgical procedure, thereby reducing time, labor and cost of treatment. Further in vivo studies would be the next step towards realizing this goal.

\section{Acknowledgements}

We gratefully acknowledge Irina Tabatadze and Julie O'Neill for processing frozen H\&E histopathology. 